\title{
Communication Architecture, Technologies, and Requirement for Modern Energy Systems
}

\author{
Muhammad Kashif*, M.J. Hossain, Yuba Raj Kafle, Edstan Fernandez, S. M. Nawazish Ali and Vivek Sharma \\ School of Engineering, Macquarie University, Sydney \\ Email: *muhammad.kashif@hdr.mq.edu.au
}

\begin{abstract}
A smart grid is an efficient and reliable means of power delivery to the end user that integrates various communication systems. The role of communication in a smart grid is crucial. The smart grid can be considered as an evolved modern grid having high reliability, efficiency and two-way communications between end users and the utility. It consists of proper communication devices which serve both utility and the end user. Moreover, it gives the opportunity for large scale integration of renewable energy, electric vehicles, and various other products and services. It combines digital intelligence, communication and advanced metering solution deployment in devices and services. Communication is an integral part that can facilitate real-time billing, maintenance, control and optimization of electricity usage.Various communication networks can be implemented in the smart grid. The main aim of this paper is to present the contemporary communication scenario in the smart grid in terms of technology, devices, network architecture and requirements in transmission and distributed energy systems.
\end{abstract}

\section{INTRODUCTION}

The traditional grid uses fossil fuels to generate the majority of its energy, which has a negative impact on environment due to greenhouse emissions [1]. Besides, it cannot address today's issues such as the increased demand of energy for the growing population, minimizing costs while maximizing system reliability and stability, global climate change, selfrecovery for equipment failure and resilience. Moreover, the traditional grid does not account for power disturbances and outages, which is a severe issue in the power system [2]. Also, due to aging, the traditional grid system is more prone to failures which may lead to blackouts which cannot be predicted.The use of renewable energy resources can be a sustainable way to deal with greenhouse gas emissions and to meet the future electricity demand [3]. However, incorporating renewable energy systems on a large scale imposes major challenges such as complex control of distributed energy resources and the storage system to meet the required capacity [4]. To provide a modern and sustainable electric-energy system, an improved technology called the smart grid has emerged [2], [5].

The smart grid improves the efficiency, economics, reliability and sustainability of the overall electrical power system. The smart grid accomplishes these tasks by using advanced digital technology that allows two-way communications between utility and customers with intelligent sensors along the transmission lines [6]. With the help of the smart grid there is a direct participation of the end user, and they know their consumption so that they can shift their load at off-peak times thereby reducing the peak demand at the peak hour. This will decrease their electricity bill because of dynamic electricity pricing with low rates in times of low load on the grid. Moreover, the smart grid enables the integration of renewable energy systems, plug-in electric vehicles in the distributed network. The energy sources owned by customers can be efficiently integrated with the distributed system [7]. Hence the user in the smart grid is either a consumer or a supplier of energy [8]. From the utility's perspective, it reduces the operation and management cost, controls intelligent appliances of the costumer's home or building to save energy, hence the consumer can get reliable, secure electricity at a low cost. It has a self-recovering capability and hence it quickly restores electricity after power disturbances [7].

The smart grid is enhanced with communication devices and sensors, and provides interoperability between these devices [9]. Incorporating two-way communications, the smart grid can improve power reliability and quality and enhance operation and maintenance tasks. The communication system realizes real-time data transfer, processing and exchange of information to facilitate efficient and reliable operation [10], [11]. Fig. 1 shows the architecture of the smart grid communication layer, which consists of a Home Area Network (HAN), a Neighborhood Area Network (NAN) and a Wide Area Network (WAN). To facilitate the control and communication of these smart nodes, a data concentrator and a control center are also included.

The rest of the paper is organized as follows. Section II includes the communication devices being used in the smart grid. Section III discusses the communication network. Section IV addresses some key technologies used for the smart grid communication system. Section V discusses the communication requirement in the smart grid and a conclusion is presented in Section VI.

\section{SMART-GRID COMMUNICATION DEVICES}

\section{A. Smart meter}

A smart meter is an advanced digital meter that records and reports energy usage in real time so that the consumer can have a proper idea about the energy consumption and billing in real time. Moreover, it has a remote disconnect function so it is suitable for a utility company in order to regulate the voltage of the end user. It is deployed to decrease the peak electrical demand, which helps to avoid overloading lines and to shift the energy use to off-peak periods. Some benefits of a smart meter are:

- Two-way real-time communication between the smart 


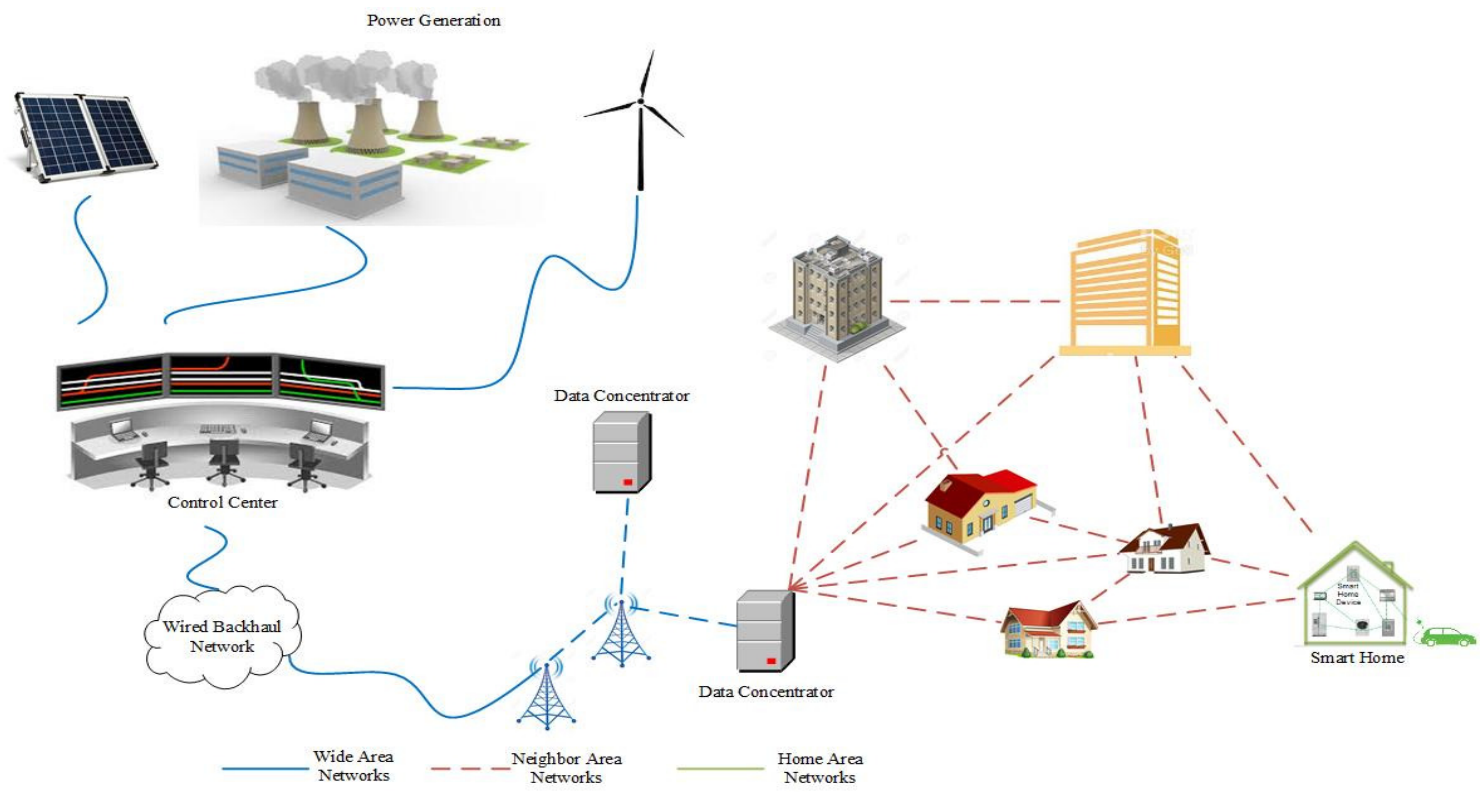

Fig. 1: Smart grid communication layer architecture

meter and the utility, which is useful for balancing electric loads

- Efficient voltage optimization and load monitoring

- Low electric cost for both the consumer and the utility company

- Demand management and direct load control

- Reducing outages and electric failures

- Self-configuring, self-healing network

- Used for AMI (Advance Metering Infrastructure) system with high security

- Reduce energy theft

- Benefit the end user in terms of energy saving, reduce carbon emission and improve service reliability.

\section{B. In-Home Display Units}

In-Home Display (IHD) provides real time powerconsumption feedback to the consumer so they can optimize energy usage to reduce their energy consumption. It is used for monitoring and controlling of energy usage by providing sophisticated LCD displays with touchscreen having wide range of user interface features. It can get consumption information through its built-in wireless sensor (RF, WiFi) or PLC. The consumption readings are stored within the unit which allows the user to get current and previous usage profiles. For the utility company it provides the consumer's information of demand response and other messages from the smart meter. It can even provide energy consumption advice by communicating with smart-grid and smart-home system. It can work together with the smart meter to realize smart energy in the HAN's of the AMI system. IHD can also support home automation.

\section{Protocol Converters}

A protocol converter is a device used for the conversion of data from one system with one protocol or standard into another protocol or standard which is compatible to another device connected to another end to achieve interoperability. It is used for the transfer of data and commands from one device to another. The protocol converter is necessary in enabling the communication in a distributed network and between different networks and protocols that co-exist in these networks. The protocol converter operates in the network layer and the conversion process will take place at the data-link layer in the smart grid. The protocol conversion process introduces delay in the system but it should be minimized in order to minimize the effect on the communication process.

\section{Data Concentrator}

The data concentrator collects and reports meter data, including data consumption, load profiles, and power-quality measurements. It also monitors and reports tampering. The collected data is sent to the server/utility via WAN using any wired or wireless IP-capable network, including DSL lines, Ethernet, CDMA, GSM, GPRS, WiMAX or optical fiber. Since it is not technically possible for all meters to communicate directly to the utility servers, data concentrators are important nodes in the AMIs, providing the interface between them. Moreover, it also detects and reports critical situations such as line breaks and device failures. It is generally installed in the street at a point in the low-voltage power-line network where the WAN signal strength is the best.

\section{SMART-GRID COMMUNICATION-NETWORK ARCHITECTURE}

Communication in the smart grid can be classified into two classes: Backbone network and access network. The backbone network includes the high-bandwidth network which uses optical fiber and digital microwave radio for the data transfer to the data concentrator, while the access network is the lowbandwidth network that normally uses copper twisted-pair 
wire lines, power-line communication and wireless systems. The communication network should however make sure to preserve the network security and the data privacy. The communication network in a smart grid can be classified into the three primary sections discussed in this paper.

\section{A. Home Area Network (HAN)}

A HAN interconnects various home appliances with the smart meter. HAN is needed to implement monitoring and control of smart devices within user premises and to implement new functionalities like demand response and AMI. HAN communication requires low cost, low bandwidth and short range, using either wired or wireless communication between smart meters and home electrical appliances. ZigBee and WiFi are usually suitable for the HAN network.

\section{B. Neighborhood Area Network (NAN)}

A NAN connects smart meters from the household premises to the data concentrator. The main function of the NAN is to gather information from the smart meter to the data concentrator. It is deployed using different communication technologies. However ZigBee and $\mathrm{WiFi}$ are predominantly used since they have low cost. Use of cellular networks like GPRS and $3 \mathrm{G}$ is also possible.

\section{Wide Area Network (WAN)}

A WAN connects the devices in the distributed substation and is responsible for the backhaul communication. The data collected from the data concentrator is transported to the distributed device, and enterprise servers are carried through the WAN network. Communication with the power plant, distribution substation and transmission lines is also carried out through the WAN. WANs are implemented using hybrid communication technology including wired and wireless technology. Since it has to handle a huge data flow, wire-line wideband communication like optical-fiber communication and wireless communication like WiMAX is usually suitable for WAN deployment, ensuring high efficiency, reliability and low latency.

\section{SMART-GRID COMMUNicATION TECHNOLOGY}

Various devices are used for efficient and reliable twoway communication between the utility grid and the smart meter. Key factors to be addressed are cost, speed, life cycle, and reliability. High speed two-way, fully integrated communication technology makes the grid a dynamic, interactive "mega-infrastructure" for real-time data and power exchange. The communication media for the communication technology include electrical power line, optical fiber, wireless, and dedicated wired network as in Fig. 2.

\section{A. Power-Line communication}

Power Line Communication (PLC) uses the existing electrical line infrastructure and provides a cost-effective approach of two-way data communication for introducing intelligent monitoring and control to the industrial approach. Since the power lines are already there, the PLC deployment is less costly distributed transmission network. Moreover, this technology supports AMI and Automatic Meter Reading (AMR) applications. In a general PLC network, Smart meters are connected to the data concentrator through power lines while data is transferred to the data center through various media generally wireless (WiFi, WiMAX) and cellular network technologies (GSM, GPRS, and OFDMA). Depending up on the power wiring characteristics, different types of PLCs use different frequency bands. For some applications, the PLC is implemented with low-frequency narrowband, while some applications utilize the wideband PLC network. A narrowband communication over power lines operating in the audio/lowfrequency band achieves data rate ranging from a few bits per second to a few kilobits per second. Therefore this is used for simple information exchange such as control of the distribution grid and for automatic remote meter reading. A broadband PLC system achieves data rate of up to $200 \mathrm{Mb} / \mathrm{sec}$ operating with the high frequency band 2-30 $\mathrm{MHz}$ [12]. However, broadband over power line has a higher data rate at the expense of coverage. Hence for a utility company, where coverage is more important than data rate, narrowband PLC is preferred to cover more difficult-to-reach locations [13]. In [13], the evolution of first-generation low-rate one-way system using ripple control to a third-generation high-rate bi-directional PLC using OFDMA technology is discussed. The second-generation PLC systems support the higher data rate with bi-directional communication and are used for distribution automation applications such as closed-loop control and AMR. The modern high-data-rate third generation system supports central load control, AMR and real-time distributionautomation applications. Various comprehensive standards are made for narrow-band and broadband power-line communication such as PRIME, PLC G3, IEEE P1901.2, ITU-T G.HNEM etc.

B. ZigBee

ZigBee is a radio communication standard which is simple and operates with low power, low cost and a low data rate. It is appropriate for the sensor network and is suitable for smart-grid applications for real-time system monitoring, load control, home automation and control, as well as building automation. They are often used in mesh networks providing high reliability and a more extensive range which allows ZigBee networks to be self-organizing ad-hoc digital radio networks. Moreover, operating in a mesh network makes it a robust wireless network which can provide excellent inbuilding coverage and support basic metering. The ZigBee is allocated in the frequency band of $2.4 \mathrm{GHz}$ and limited to a data rate of $250 \mathrm{~kb} / \mathrm{s}$. This frequency band is the same as the frequency band used by wireless local-area-network (WLAN) channels or WiFi networks so it suffers with interference. In [14] the author proposes ZigBee deployment guidelines under interference of WLAN, identifying "Safe Distance" and "Safe Offset Frequency" using a comprehensive approach including theoretical analysis, software simulation, and empirical measurement. ZigBee can be combined with PLC for exchanging data between utilities and smart metering devices and appliances located on customer premises [15]. It can support devices like Programmable Communicating Tstats (PCTs), Load-Control Devices, In- Home Displays, Energy- 


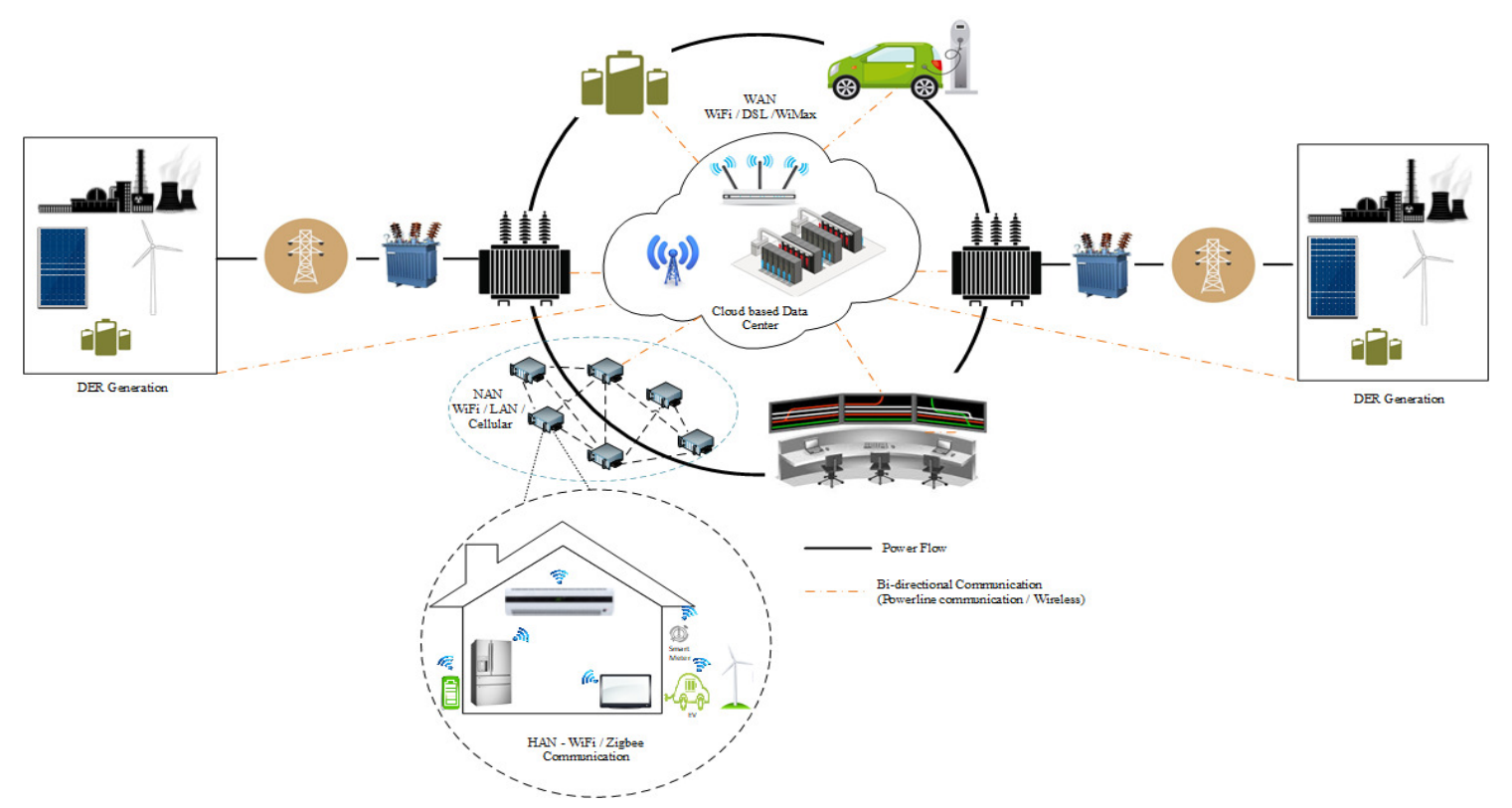

Fig. 2: Communication Technologies in Smart Grids

Management Systems [16]. Moreover, it is highly secure as it incorporates an out-of-band step for an added layer of security for utility as well as consumer [17]. The ZigBee network is based on IEEE 802.15 .4 standards defined by the U.S. National Institute for Standards and Technology. ZigBee has a major deployment in the USA, Canada, Sweden and Australia.

\section{Wireless mesh network}

A wireless network transfers information in the air medium. Various wireless networks can be deployed for smart-grid communication for transferring data from smart meter to the data concentrator and vice versa. Also wireless can be deployed for the communication between the data concentrator and the data center. Many wireless communications like GSM, GPRS, WCDMA, WiMAX and WiFi can be used as the wireless network. Since we are using a third-party's wireless networks, infrastructure development for the wireless communication is not required, which decreases the initial installation cost. A wireless mesh network provides wireless coverage over a wider range and consists of a group of nodes. Each node can act as an access point, sending and receiving data over a mesh network. If any device fails then it updates to the next route via the active node. In a smart grid, a smart meter could be the access point connected with the aggregation node to the local substation and finally to the utility through a WAN. The data from the customer is collected by the smart meter and is forwarded to the data concentrator through the mesh network, from where it makes its way to the utility data center through some broadband communication network [18]. A wireless mesh network is inexpensive, high-speed and easy to implement. Wireless mesh network is usually based on 802.11 protocols. In PG\&E's smart meter system, each smart device in the house premises are equipped with a radio module which routes the metering data via nearby meters [6].

\section{Cellular network}

The cellular network can be used for communicating the data from the smart meter to the data concentrator across NAN [19]. The cellular network now has evolved from the second-generation (2G) network called Global System for Mobile (GSM) which used GPRS as a data service. Utilities have primarily used 2G, 2.5G, 3G, Long Term Evolution (LTE) and WiMAX technology. However, most of the cellular network which is used as the smart metering infrastructure has evolved as a part of GSM, which uses GPRS as a data service. Enhanced Data rates for GSM Evolution (EDGE), that allows improved data transmission rates, is considered as a pre-3G radio technology, having better capacity and performance than a GPRS connection. EDGE can be considered as $2.5 \mathrm{G}$ which has bandwidth up to $236 \mathrm{kbit} / \mathrm{s}$ and is four times as efficient as GPRS and so more device can be connected over the network.

For data communication with a large coverage and a high data rate WiMAX, 3G or 4G cellular technology can be deployed. WiMAX has high bandwidth (up to $1 \mathrm{Gbit} / \mathrm{s}$ ) and large coverage range while the $3 \mathrm{G}$ service, with data rates of at least $200 \mathrm{kbit} / \mathrm{s}$, has better spectral efficiency and speed than $2 \mathrm{G}$. There are two distinct 3G families: 3GPP and 3GPP2. 3GPP technology evolved as GPRS (144 Kbps), EDGE (384 kbps), WCDMA (1.92 Mbps), HSDPA (14Mbps), and E-UTRA (aiming for $100 \mathrm{Mbps})$. 3GPP2 uses CDMA2000 transition to 3G which evolved as 1xRTT (144 Kbps), EV-DO (2.4 Mbps), EVDO Rev. A (3.1 Mbps), EV-DO Rev. B (4.9 Mbps), and UMB (aiming to reach $288 \mathrm{Mbps}$ ). LTE and Ultra Mobile Broadband (UMB) are often called fourth-generation technology because of the downlink speed. 4G provides ultra-broadband data and is commercially deployed in South Korea as WiMAX standard and in Norway with the first-release LTE standard. Hence for the advanced metering infrastructure deployment, 
the supplier has more choice for the data service in terms of cost, coverage and speed of the data service. In the USA, the smart-meter- maker company Echelon Corp and the cellularservice provider T-Mobile USA joined for the communication by incorporating T-Mobile's SIM to Echelon's smart meters. Echelon installed cellular radio modules in its smart meters which makes them communicate with one another through the power line and links the data to and from utilities by using T-Mobile's network. Also, partnerships including those between smart-grid vendor SmartSynch and AT\&T's wireless network serve as a communication platform between smart meters and the utility in the USA. The $4 \mathrm{G}$ protocols provide enhanced bandwidth, security, data transfer rate and technical advancement over previous generations of protocols. Hence 4G technology is best suited for the NAN implementation in the smart grid. [20] proposes an architecture for integrating renewable energy-powered cellular networks and the Smart Grid based on technology requirements, which brings mutual benefits for the Smart grid and network operators.

\section{E. Digital Subscriber Lines}

Digital Subscriber Lines (DSLs) facilitate high-speed digital data communication by the use of existing PSTN lines. DSL line is a point-to-point circuit-switched network having dedicated connectionS, which provides the backhaul for smart grid data from homes to the utility. The smart grid can coordinate with the telephone company by using their DSL line. There are three different types of DSL depending upon the speed namely: Asymmetric DSL (ADSL), High-speed DSL (HDSL), and Very High Data Rate DSL (VDSL). However, the throughput depends on the distance from the telephone exchange to the end user. When the distance increases then it will increase the signal attenuation and decrease the signalto-noise ratio. A comparison of smart grid communication technologies with their advantages and disadvantages is shown in Table.I.

\section{SMART GRID COMMUNICATION REQUIREMENT}

The smart grid needs an interaction between generation, transmission and distribution networks, which requires monitoring/sensing, communication and control among them to meet the total grid requirement. Because of continually changing technology this incorporates different communication technologies on different networks that fits to all its needs. Requirements of high bandwidth, low latency, robustness, interoperability, security are some major considerations.

- Secure: Since there is a two-way communication between the centralized software and millions of smart devices throughout the electrical network, the smart grid is vulnerable to a cyber-attack.

- Reliable and robust: The aging of the power infrastructure, the peak demands and the increasing volume of energy consumption are the main issues that are responsi-

TABLE I: Comparison of smart grid communication technologies

\begin{tabular}{|c|c|c|}
\hline $\begin{array}{c}\text { Communication } \\
\text { technologies }\end{array}$ & Advantages & Disadvantages \\
\hline$P L C$ & $\begin{array}{l}\text { - Less installation cost due to already available } \\
\text { power infrastructure for data communication } \\
\text { - Efficiently works where RF cannot }\end{array}$ & $\begin{array}{l}\text { - Severe EMI due to unshielded power-line ca- } \\
\text { bles } \\
\text { - High attenuation and noise on power lines } \\
\text { - Low security and channel distortion }\end{array}$ \\
\hline ZigBee & $\begin{array}{l}\text { - Low power consumption allows the use of } \\
\text { smaller batteries with longer life and makes } \\
\text { it suitable for wireless sensor network } \\
\text { - Easier and faster installation } \\
\text { - Highly scalable and inter-operable } \\
\text { - Robust and highly secure }\end{array}$ & $\begin{array}{l}\text { - ZigBee uses the same frequency band as Wi- } \\
\text { Fi and WLAN, so suffers interference, causing } \\
\text { performance degradation }\end{array}$ \\
\hline Wireless mesh network & $\begin{array}{l}\text { - Wireless nodes are self-configuring, self- } \\
\text { tuning and self-healing, which makes the mesh } \\
\text { network scalable, reliable, intelligent and high } \\
\text { speed } \\
\text { - low-cost network with load balancing capabil- } \\
\text { ity, better coverage and scalable capacity } \\
\text { - It works with multi-hop routing and line of } \\
\text { sight is not required }\end{array}$ & $\begin{array}{l}\text { - The nodes which are not ad hoc are prone to } \\
\text { hackers, a big threat from a security perspec- } \\
\text { tive }\end{array}$ \\
\hline Cellular Network & $\begin{array}{l}\text { - Cost-effective, High data rate, wide coverage } \\
\text { range, scalable, flexible } \\
\text { - Utility can use already available Cellular net- } \\
\text { works which save the cost of additional infras- } \\
\text { tructure }\end{array}$ & $\begin{array}{l}\text { - Cost-effective, High data rate, wide coverage } \\
\text { range, scalable, flexible } \\
\text { - Utility can use already available Cellular net- } \\
\text { works which save the cost of additional infras- } \\
\text { tructure }\end{array}$ \\
\hline Digital Subscriber line & $\begin{array}{l}\text { No installation cost for utility companies due } \\
\text { to availability of existing telephone }\end{array}$ & $\begin{array}{l}\text { - With the increase of distance DSL suffer a high } \\
\text { bit error rate } \\
\text { - Using DSL for the communication should re- } \\
\text { quire having a DSL line but not everyone has } \\
\text { a DSL connection }\end{array}$ \\
\hline
\end{tabular}


ble for the unreliability of the power grid. In order to get high reliability and robustness, combined communication networks with wired and wireless abilities are the best option.

- Flexible and Scalable: The smart grid should be flexible enough to accommodate any advancement in the system so that it can be upgraded with a slight modification. It needs the scalability of accommodating more devices and services into entire grid area and should be easily expandable. With a small modification in the system, it should be fully capable of acquiring feature enhancements and support multi-application requirements from demand-side management to distribution automation.

- Cost-effective solution: The smart grid should be costeffective for both the consumer and the utility company. By using the existing communication infrastructure, the deployment of a smart grid will be cost-effective. It optimizes the balance of power supply and demand so that the peak demand will be lower. This leads to a reduction of the operational cost of smart grid.

- Interoperability: This is the ability of two different devices, networks, systems, application or components to work together and exchange information among the interconnected elements of the smart grid system. In a smart grid smart devices from various vendors are used. Interoperability allows interactions between the vast number of interconnected devices and systems having different architecture, technology and software.

- Quality of service: The smart grid should guarantee Quality of Service (QOS) for high-performance communication infrastructure and networking technology in power generation, transmission and distribution networks avoiding possible disruptions and blackouts. The bandwidth and the latency are two critical QOS factors that need to be considered for a better QOS requirement in smart grids.

\section{CONCLUSION}

The smart grid is an intelligent power generation, distribution and control system with digital two-way communication and supporting real-time system monitoring, load control and home automation. The communication network in the smart grid is essential to make the smart grid automated, reliable, self-healing, secure, manageable, controllable, flexible, integrated and observable. The choice of communication topology, media and devices decides the performance, cost and implementation issues in deployment of the smart grid. This paper provides a brief review of various aspects related to communication network architecture. Several communication technologies along with their pros and cons are discussed. The communication devices and sensors necessary to realize data collection, transfer and processing are stated. Finally, various performance criteria that aid in comparison of several communication architectures, devices and technologies are presented.

\section{REFERENCES}

[1] Worldenergy.org, 'World Energy Resources: 2013 Survey', 2015.[Online]. Available:https://www.worldenergy.org/publications/2013/worldenergy-resources-2013-survey. [Accessed: 09- Nov- 2015].

[2] Fang, Xi, Satyajayant Misra, Guoliang Xue, and Dejun Yang. "Smart grid-The new and improved power grid: A survey." IEEE communications surveys \& tutorials 14, no. 4 (2012): 944-980.

[3] Sims, Ralph EH, Hans-Holger Rogner, and Ken Gregory. "Carbon emission and mitigation cost comparisons between fossil fuel, nuclear and renewable energy resources for electricity generation." Energy policy 31, no. 13 (2003): 1315-1326.

[4] Huang, Alex Q., et al. "The future renewable electric energy delivery and management (FREEDM) system: the energy internet." Proceedings of the IEEE 99.1 (2011): 133-148.

[5] Sarwar, Muhammad, and Bilal Asad. "A review on future power systems; technologies and research for smart grids." In Emerging Technologies (ICET), 2016 International Conference on, pp. 1-6. IEEE, 2016.

[6] Gungor, Vehbi C., Dilan Sahin, Taskin Kocak, Salih Ergut, Concettina Buccella, Carlo Cecati, and Gerhard P. Hancke. "Smart grid technologies: Communication technologies and standards." IEEE transactions on Industrial informatics 7, no. 4 (2011): 529-539.

[7] Farhangi, Hassan. "The path of the smart grid." IEEE power and energy magazine 8, no. 1 (2010).

[8] Yan, Ye, Yi Qian, Hamid Sharif, and David Tipper. "A survey on smart grid communication infrastructures: Motivations, requirements and challenges." IEEE communications surveys \& tutorials 15, no. 1 (2013): $5-20$.

[9] Kafle, Y. R., K. Mahmud, S. Morsalin, and G. E. Town. "Towards an internet of energy." In Power System Technology (POWERCON), 2016 IEEE International Conference on, pp. 1-6. IEEE, 2016.

[10] Fan, Zhong, Parag Kulkarni, Sedat Gormus, Costas Efthymiou, Georgios Kalogridis, Mahesh Sooriyabandara, Ziming Zhu, Sangarapillai Lambotharan, and Woon Hau Chin. "Smart grid communications: Overview of research challenges, solutions, and standardization activities." IEEE Communications Surveys \& Tutorials 15, no. 1 (2013): 21-38.

[11] Gungor, V. Cagri, Dilan Sahin, Taskin Kocak, Salih Ergut, Concettina Buccella, Carlo Cecati, and Gerhard P. Hancke. "A survey on smart grid potential applications and communication requirements." IEEE Transactions on industrial informatics 9, no. 1 (2013): 28-42.

[12] Galli, Stefano, Anna Scaglione, and Zhifang Wang. "For the grid and through the grid: The role of power line communications in the smart grid." Proceedings of the IEEE 99, no. 6 (2011): 998-1027.

[13] Dzung, Dacfey, Inigo Berganza, and Alberto Sendin. "Evolution of powerline communications for smart distribution: From ripple control to OFDM.” In Power Line Communications and Its Applications (ISPLC), 2011 IEEE International Symposium on, pp. 474-478. IEEE, 2011.

[14] Yi, Peizhong, Abiodun Iwayemi, and Chi Zhou. "Developing ZigBee deployment guideline under WiFi interference for smart grid applications." IEEE transactions on smart grid 2, no. 1 (2011): 110-120.

[15] Han, Jinsoo, Chang-Sic Choi, Wan-Ki Park, Ilwoo Lee, and Sang-Ha Kim. "Smart home energy management system including renewable energy based on ZigBee and PLC." IEEE Transactions on Consumer Electronics 60, no. 2 (2014): 198-202.

[16] Batista, N. C., R. Melício, J. C. O. Matias, and J. P. S. Catalão. "Photovoltaic and wind energy systems monitoring and building/home energy management using ZigBee devices within a smart grid." Energy 49 (2013): 306-315

[17] Fan, Bo. "Analysis on the Security Architecture of ZigBee Based on IEEE 802.15. 4." In Autonomous Decentralized System (ISADS), 2017 IEEE 13th International Symposium on, pp. 241-246. IEEE, 2017.

[18] Robertsingh, A., D. Devaraj, and R. Narmathabanu. "Development and analysis of wireless mesh networks with load-balancing for AMI in smart grid." In Computing and Network Communications (CoCoNet), 2015 International Conference on, pp. 106-111. IEEE, 2015.

[19] Kalalas, Charalampos, Linus Thrybom, and Jesus Alonso-Zarate. "Cellular communications for smart grid neighborhood area networks: A survey." IEEE Access 4 (2016): 1469-1493.

[20] Hassan, Hussein Al Haj, Alexander Pelov, and Loutfi Nuaymi. "Integrating cellular networks, smart grid, and renewable energy: Analysis, architecture, and challenges." IEEE access 3 (2015): 2755-2770. 